\title{
Combined Integrating Control for High Order System with Time-delay
}

\author{
Angang Chen ${ }^{1,}$, , Zhengyun Ren ${ }^{2}$ b \\ ${ }^{1}$ School of information science and technology, Donghua University, Shanghai 201620, China; \\ ${ }^{2}$ School of information science and technology, Donghua University, Shanghai 201620, China; \\ adhu_chenangang@163.com, ${ }^{\mathrm{b}}$ renzhengyun@dhu.edu.cn
}

Keywords: Model reduction, High order processes with time-delay, combined integrating controller.

\begin{abstract}
For complex, high-order processes with time-delay, this paper proposes a model reduction method based on a numerical optimization algorithm, and design combined integrating controller based on the reduced model, the controller is applied to the original model can get a good control effect. Model reduction method makes high order processes with a time - delay approximation for the first order delay object or second order delay object, through step response and Bode chart compares, the curve of the reduced model can approximate the original curve model. Combined integrating control is based on the model of the controller, has a good control effect on processes with time-delay, model reduction algorithm makes the combined integrating controller good application to complex higher- order processes with time-delay, and its structure is simple, less tunable characteristics.
\end{abstract}

\section{Introduction}

In the actual process of industrial objects, the controlled objects are mostly high order system with time-delay, which often did not achieve very good results; the control of such systems has always been studied by scholar industrial hotspots. For complex, high order systems with time-delay, General designed with two control methods: model order reduction and direct design controllers, but the latter method of controller structure is complex, and various parameters, implemented in the actual difficulties. The model order reduction made approximate low-order objects, structure design of the controller will be a lot of simplified.

This paper presents an optimal model reduction algorithms for high order system with time-delay, the first high order system with time-delay are approximated into higher-order model without time-delay by Padé approximation algorithm, through numerical optimization model reduction into the first order delay object or second order delay object ${ }^{[1]}$, so that designers can replace the original model with a reduced-order model to design controller, then the controller used in the original model. The controller used in this paper is a combined integral controller, the combination integral controller to control the low-order time- delay without overshoot, quick response, simple structure, less adjustable parameters.

\section{The frequency domain optimal model order reduction algorithm}

In a high order system with time delay controller design and analysis, the model reduction first in 1966 by Edward Davison ${ }^{[2]}$, after decades of development, a variety of reduced-order algorithm, mainly by the Padé ${ }^{[3]}$ and Routh ${ }^{[4]}$ algorithm. Padé algorithm widely used in time domain and frequency domain, Padé algorithm thoughts reserved former vector are $+k+1$ time, but it cannot guarantee the stability of the reduced-order model and Routh algorithm, greatest feature is stable after reduction of the system to ensure its stability reduced-order model, But the effect is not very ideal fitting.

\subsection{Reduced order model}

The original model for the high order system time-delay is

$$
G(s)=\frac{b_{1} s^{n-1}+\cdots+b_{n-1} s+b_{n}}{s^{n}+a_{1} s^{n-1}+\cdots+a_{n-1} s+a_{n}} e^{-l s}
$$


Padé algorithm high order approximation model

$$
G_{r}(s)=\frac{p_{1} s^{n+r-1}+\cdots+p_{n+r-1} s+p_{n+r}}{s^{n+k}+q_{1} s^{n+k-1}+\cdots+q_{n+k-1} s+q_{n+k}}(r<k)
$$

According to the error signal e (t) to define $J_{I S E}=\int_{0}^{\infty} e^{2}(t) d t$, this is defined as the objective function, to minimize, to get the optimal reduced order model.

$$
G(s)
$$

Padé approximation for higher-order

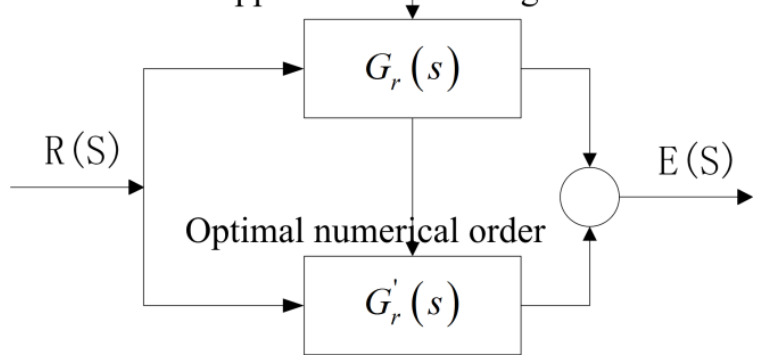

Fig. 1 Model order reduction

The low order delay model is obtained by the numerical optimization algorithm

$$
G_{r}^{\prime}(\mathrm{s})=\frac{\beta_{1} s^{r}+\cdots+\beta_{r} s+\beta_{r+1}}{s^{k}+\alpha_{1} s^{k-1}+\cdots+a_{k-1} s+a_{k}} e^{-\tau s}
$$

Reduced order error signal Laplace transforms expression

$$
E(\mathrm{~s})=\left[G_{r}(\mathrm{~s})-G_{r}^{\prime}(\mathrm{s})\right] \mathrm{R}(\mathrm{s})
$$

\subsection{Rule of error signal}

$\mathrm{R}(\mathrm{s})$ is the Laplace transform of the input signal, the high order process $\mathrm{Gr}(\mathrm{s})$ and reduction process $\mathrm{Gr}^{\prime}(\mathrm{s})$ of error is $\mathrm{E}(\mathrm{s})$, define a new error signal $\mathrm{h}(\mathrm{t})=\mathrm{w}(\mathrm{t})$ e $(\mathrm{t})$, then define a new rule of ISE.

$$
\sigma_{h}^{2}=\int_{0}^{\infty} \mathrm{h}^{2}(t) d t=\int_{0}^{\infty} \mathrm{w}^{2}(t) e^{2}(t) d t
$$

For a given input signal and $\theta=\left[\alpha_{1}, \alpha_{2}, \cdots, \alpha_{\mathrm{m}}, \beta_{1}, \beta_{2}, \cdots \beta_{r+1}, \tau\right]$ can define a reduced order model's error signal e $(\mathrm{t}, \theta)$, the error signal into $\theta$ functions, so that we can define an optimal objective function of the reduced order

$$
J=\min _{\theta}\left[\int_{0}^{\infty} \mathrm{w}^{2}(t) e^{2}(t, \theta) d t\right]
$$

This method is based on the same input signal, original models and reduced order model error variances weighted integral to a minimum, this method by numerical optimization algorithms to calculate.

\section{Combination of integral control algorithm}

In practical projects in the industry, through the output data modeling process and mechanism analysis of a transfer function is ${ }^{[5]}$ :

$$
\begin{gathered}
G_{P}(s)=\frac{k}{\tau s}\left(1-e^{-\tau s}\right) \\
G_{P}(s)=\frac{k}{\tau_{1} s}\left(1-e^{-\tau_{1} s}\right) e^{-\tau_{2} s} \\
G_{P}(s)=\frac{k}{\tau s(T s+1)}\left(1-e^{-\tau s}\right) \\
G_{P}(s)=\frac{k}{\tau_{1} s(T s+1)}\left(1-e^{-\tau_{1} s}\right) e^{-\tau_{2} s}
\end{gathered}
$$




$$
G_{P}(s)=\frac{k_{1}}{\tau_{1} s}\left(1-e^{-\tau_{1} s}\right) e^{-\tau_{2} s}+\frac{k_{2}}{\tau_{3} s}\left(1-e^{-\tau_{3} s}\right) e^{-\tau_{4} s}, \tau_{4}=\tau_{1}+\tau_{2}
$$

This type of transfer function is a superposition of multiple time-delay integration objects, and has a $\left(1-e^{-\tau s}\right)$ link; we call it a combined integrating system. Such transfer function has not left the plane of the pole, but also has not left the plane of zero; Look for the characteristic, this kind of object has both integral characteristics, but also has a non-minimum phase property. Combined integrating object in essence is a kind of open-loop stable system; combination integral controller is presented based on the characteristic.

The combination of the integrated control algorithm is developed on the basis of model predictive control, an advanced control algorithm. Designed controller based on the object model and the desired combination integral function, so this type of controller design only needs to know that the object model to design the controller.

\subsection{Algorithm theory}

General negative feedback control system is shown in Figure 2:

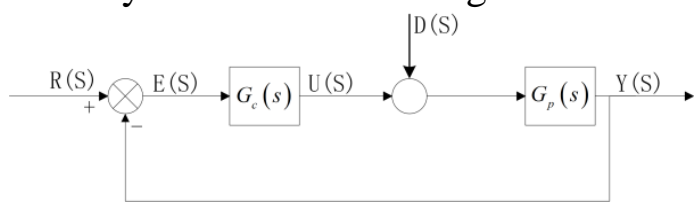

Fig. 2 Negative feedback control system

Combination of the integral control algorithm is designed based on the object model, by the object model and the desired closed-loop transfer function to get the controller. Assuming that the desired closed loop transfer function of a combination of integral object.

$$
\begin{gathered}
G_{0}(s)=\frac{1}{\tau_{1} s}\left(1-e^{-\tau_{1} s}\right) e^{-\tau_{2} s} \\
G_{0}(s)=\frac{1}{\tau_{1} s(T s+1)}\left(1-e^{-\tau_{1} s}\right) e^{-\tau_{2} s}
\end{gathered}
$$

Because the combination of integral nature is open-loop stable, its dynamic response curve rise process does not produce overshoot, is very important to the stability of the system. Algorithm including one variable parameter $\tau 1, \tau 1$ is smaller, the faster the system response speed, in the actual control system, generally the $\tau 1$ approximation for the system of delay time $\tau 2$.

\subsection{Reduced-order model combination integral controller}

For high order systems with time-delay model order reduction is simplified to a first order delay object model, can reflect the dynamic characteristics, the first order delay object model transfer function is ${ }^{[6]}$ :

$$
G_{P}(s)=\frac{k}{T s+1} e^{-\tau s}
$$

A high order system with time-delay order model reduction to get a first order delay object model (14), the controller is designed according to this model. Assuming that the desired closed-loop transfer function is:

$$
\mathrm{G}_{0}(\mathrm{~s})=\frac{1}{\tau_{1} s}\left(1-e^{-\tau_{1} s}\right) e^{-\tau s}
$$

$\tau 1$ as adjustable parameters, $\tau 1$ is smaller, faster the closed loop system response, conversely the more slowly.

That controller transfer function

$$
G_{c}(s)=\frac{(T s+1)}{k \tau_{1} s}\left(1-e^{-\tau_{1} s}\right) \cdot \frac{1}{1-\frac{1}{\tau_{1} s}\left(1-e^{-\tau_{1} s}\right) e^{-\tau s}}
$$


For high order of oscillation process and non-minimum phase link, order model reduction to get the second order delay object model to reflect the dynamic characteristics, the second order delay object model transfer function is:

$$
G_{P}(s)=\frac{k}{\left(T_{1} s+1\right)\left(T_{2} s+1\right)} e^{-\tau s}
$$

Assuming the desired closed-loop transfer function is

$$
G_{0}(s)=\frac{k}{\tau_{1} s}\left(1-e^{-\tau_{1} s}\right) e^{-\tau s}
$$

$\tau 1$ meaning again, namely the transfer function of controller

$$
G_{c}(s)=\frac{\left(T_{1} s+1\right)\left(T_{2} s+1\right)}{k}\left(1-e^{-\tau_{1} s}\right) \cdot \frac{1}{1-\frac{1}{\tau_{1} s}\left(1-e^{-\tau_{1} s}\right) e^{-\tau s}}
$$

\section{The simulation research}

Consider the oscillation of higher-order systems with time delay

$$
G(s)=\frac{(\mathrm{s}+1)^{2}}{\left(s^{2}+\mathrm{s}+1\right)^{2}(s+2)^{4}} e^{-2 s}
$$

First by the Padé approximation method to get higher order model (21), then by numerical optimal model order reduction to get a standard second order time delay model (22).

$$
\begin{gathered}
G_{r}(s)=\frac{s^{4}-s^{3}-2 s^{2}+3 s+3}{s^{10}+13 s^{9}+76 s^{8}+265 s^{7}+616 s^{6}+1009 s^{5}+1195 s^{4}+1024 s^{3}+616 s^{2}+240 s+48} \\
G_{r}^{\prime}(s)=\frac{0.0625}{1.3841 s^{2}+0.7366 s+1} e^{-3.39 s}
\end{gathered}
$$

Step response for reduced order model and the original model and their Bode diagram as shown in figure 3 and 4, as can be seen from the step response diagram Padé approximation of the high order model to better approximate the original model, higher order model reduced by numerical optimization model approximate to the low order delay model can also be a good approximation to the original model, the order of the model is reduced greatly, and the degree of approximation to conform to the requirements of the system. In the Bode diagram can be seen in the low frequency curve fitting degree is high, in the high frequency appears difference is very big, but the unit is the $\mathrm{dB}$, so in the high frequency is not different. Based on the reduced order model (22) design combined integrating controller, according to the type (19) to evaluate the controller transfer function, by adjusting the adjustable parameter $\tau 1$ to adjust the system response speed. Then the controller applied to the original system as in Figure 5, it can be seen that Based on reduced order model design combined integrating controller fully adapted to the original model, and at $\mathrm{t}=40 \mathrm{~s}$ to join the unit step disturbance, its control effect is still good.

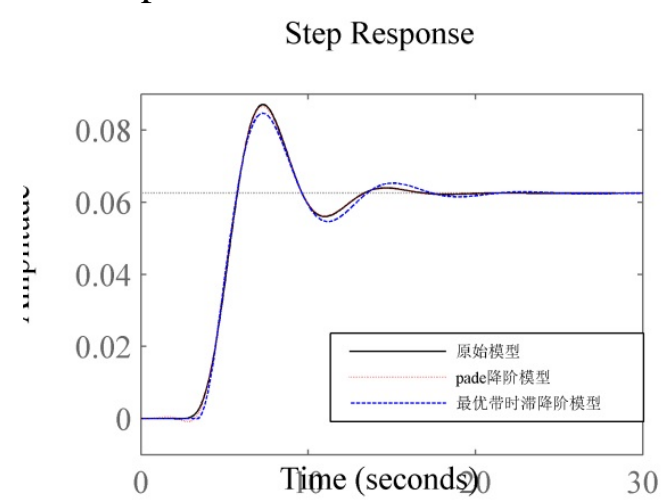

Fig. 3 Comparisons of step response curve

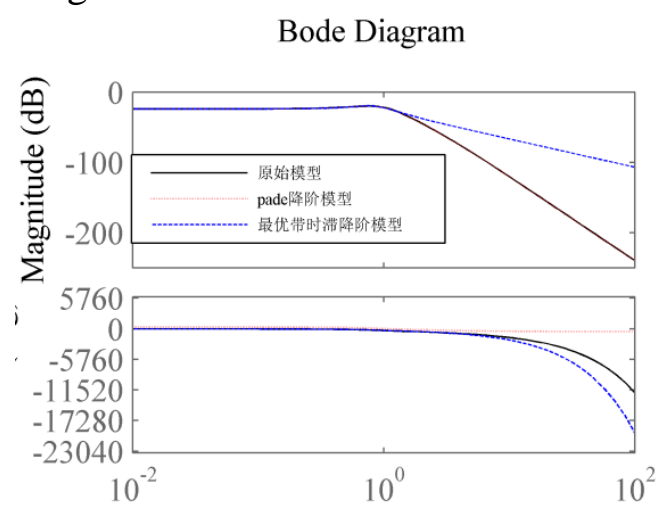

Fig. 4 Comparisons of Bode diagram 


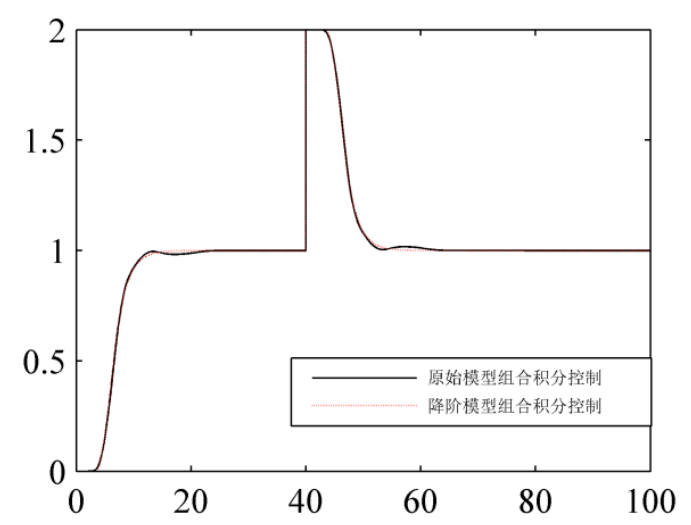

Fig. 5 Comparisons of combined integrating control

Considering non-minimum phase high order with time-delay model

$$
G(s)=\frac{10 s^{3}-60 s^{2}+110 s+60}{s^{4}+17 s^{3}+82 s^{2}+130 s+100} e^{-s}
$$

First by the Padé approximation method to get higher order model (24), then by numerical optimal model order reduction, reduced to the second order ahead of time-delay model (25).

$$
\begin{gathered}
G_{r}(s)=\frac{10 s^{5}-120 s^{4}+590 s^{3}-1320 s^{2}+960 s+720}{s^{6}+23 s^{5}+196 s^{4}+826 s^{3}+1864 s^{2}+2160 s+1200} \\
G_{r}^{\prime}(s)=\frac{1.4207 s+0.5999}{0.4198 s^{2}+0.9685 s+1} e^{-1.84 s}
\end{gathered}
$$

Step response for reduced order model and the original model and their Bode diagram as shown in figure 6 and 7, as can be seen from the step response diagram Padé approximation of the high order model and numerical optimization model in the early stages are error approximation of the original model, at the start of the original model and Padé approximation of high-order model have oscillation, and numerical optimization model order reduction ignored the approximation of the initial stage, but after 2 s basically is a good way to close to the original model. In the Bode diagram can be seen in the low frequency curve fitting degree is high. Based on the reduced order model (25) design combined integrating controller, according to the type (19) to evaluate the controller transfer function, by adjusting the adjustable parameter $\tau 1$ to adjust the system response speed. Then the controller applied to the original system as in Figure 8, it can be seen that Based on reduced order model design combined integrating controller fully adapted to the original model, and at $\mathrm{t}=40 \mathrm{~s}$ to join the unit step disturbance, its control effect is still good.

Step Response

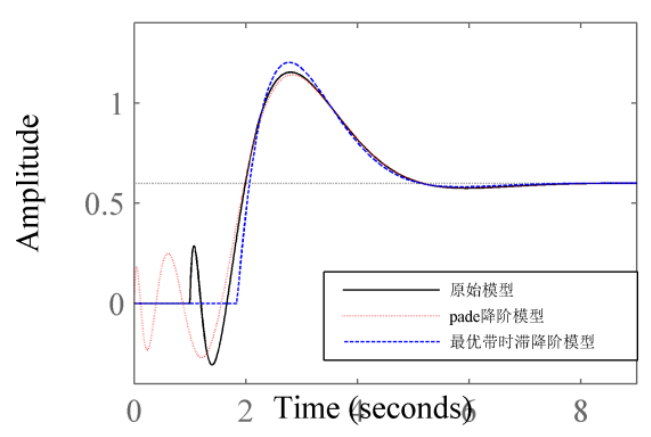

Fig. 6 Comparisons of step response curve
Bode Diagram

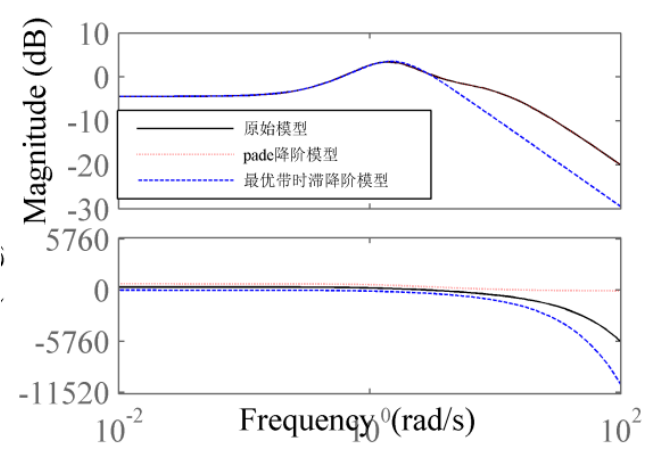

Fig. 7 Comparisons of Bode diagram 


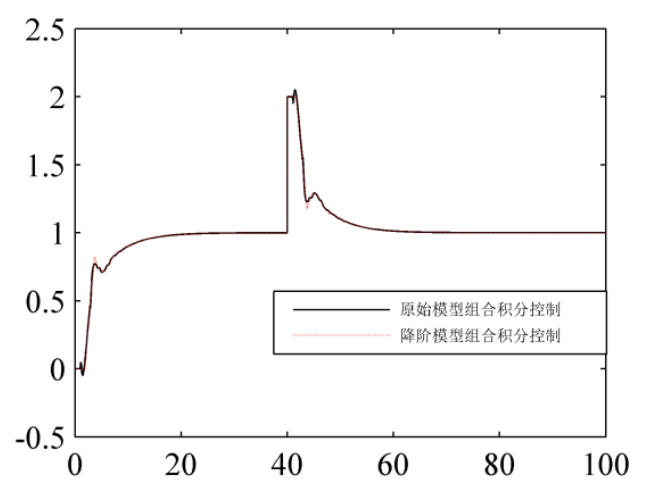

Fig. 8 Comparisons of combined integrating control

Above by simulation of high order system with time-delay object, especially for high order oscillations and high order non-minimum phase objects by Padé approximation for the high order model, then by numerical optimal model order reduction to get a standard, low order time-delay model, from step response curve and the Bode diagram, its high fitting accuracy, so based on the reduced order model design combined integrating controller fully applied to the original model can get almost the same effect.

\section{Summary}

For high order systems with time-delay, this paper presents a model order reduction algorithm based on the Padé algorithm and numerical optimization combination of integral control. First of all, the original model using the Padé approximation method to get high order model, then by numerical optimal model order reduction reduced to the low order time-delay model, the reduced order model overcame the Padé approximation of instability and poor fit of the reduced order of Routh; Secondly, the combination of integral control come from industrial real object, using its open loop stable quality, successfully applied to other complex industrial object, simple structure, less adjustable parameters, easy implementation, the simulation shows the feasibility and effectiveness of this algorithm.

\section{Acknowledgments}

This work was financially supported by research and application of combined integrating control system fund (NO.:60674088).

\section{Reference}

[1] Xue Dingyu. Control System Optimal Reduction Technique and its Applications [J]. Control and Decision, 1994, Vol.9 NO.6.

[2] Davison E J. A method for simplifying linear dynamic systems. IEEE Transaction on Automatic Control, 1966, AC-11: 93 101

[3] Bultheel A, van Barel M. Padé techniques for model reduction in linear system theory: a survey. Journal of Computational and Applied Mathematics, 1986, 14:401 438.

[4] Hutton M F.Routh approximation for high-order linear systems. Proceedings of $9^{\text {th }}$ Allerton Conference, 1971, 160 169.

[5] Ren Zhengyun, Huang Biao. Control of combined integrating systems with industrial application. International Journal of Process System Engineering. 2008, 1(1): 77-86.

[6] Zheng Da, Ren Zhengyun, Fang Jian’an. Stability analysis of multiple time delayed system. ISA Transactions. 2008, 47(4): 439-447. 